\title{
CONTEÚDO E EXPORTAÇÃO DE B, CU, FE, MN, ZN E NA EM ACÁCIA- NEGRA (ACACIA MEARNSII DE WILD.) PROCEDÊNCIA LAKE GEOGE BUNGE DORE - AUSTRÁLIA
}

\author{
Marcos Vinicius Winckler Caldeira ${ }^{1}$ \\ Rubens Marques Rondon Neto ${ }^{2}$ \\ Mauro Valdir Schumacher ${ }^{3}$ \\ Peter Spathelf ${ }^{4}$
}

\begin{abstract}
RESUMO
O presente trabalho teve por objetivo determinar o conteúdo e a exportação de $\mathrm{B}, \mathrm{Cu}, \mathrm{Fe}, \mathrm{Mn}$, $\mathrm{Zn}$ e Na, nos diferentes componentes das árvores de um povoamento de acácia-negra (Acacia mearnsii De Wild.), procedência australiana Lake Geoge Bunge Dore, com 2,4 anos de idade no município de Butiá - RS (Brasil). A biomassa total encontrada foi de $41.107 \mathrm{~kg} \mathrm{ha}^{-1}$, da qual, aproximadamente 49,$5 ; 19,5 ; 18,2 ; 11,6$ e 1,2 \%, estavam distribuídos na madeira do lenho, galhos vivos, folhas, casca e galhos mortos, respectivamente. A acumulação dos elementos na árvore ficou assim: folhas $(40,1 \%)$, galhos vivos $(18,6 \%)$, casca $(17,9 \%)$, galhos mortos $(14,3 \%)$ e madeira do tronco $(9,1 \%)$. A quantidade estimada de elementos contido na biomassa aérea foi de $10,57 \mathrm{~kg} \mathrm{ha}^{-1}$, acumulados da seguinte forma: $\mathrm{Na}(50,6 \%), \mathrm{Fe}(23,3 \%), \mathrm{Zn}(12,5 \%), \mathrm{Mn}(7,8 \%), \mathrm{B}(4,4 \%)$ e $\mathrm{Cu}(1,4 \%)$. Concluise que $73,1 \%$ dos nutrientes da biomassa acima do solo da acácia-negra, se acumularam na copa (folhas, galhos vivos e mortos).

Palavras-chave: Acacia mearnsii, acácia-negra, micronutrientes, exportação de micronutrientes e Na.

\section{CONTENT AND EXPORTATION OF B, CU, FE, MN, ZN AND NA IN BLACK WATTLE (ACACIA MEARNSII DE WILD.) AUSTRALIAN LAKE GEOGE BUNGE DORE PROCEDENCE}

\begin{abstract}
The present work had for objective to determine the content and the export of $\mathrm{B}, \mathrm{Cu}, \mathrm{Fe}, \mathrm{Mn}$, $\mathrm{Zn}$ and $\mathrm{Na}$, in the different components of the trees of a forest of black wattle (Acacia mearnsii De Wild.), from Lake Geoge Bunge Dore Australian provenance, with 2,4 years of age in the district of Butiá - RS (Brazil). The total above-biomass found was of $41.107 \mathrm{~kg} \mathrm{ha}^{-1}$, of the which, approximately 49,$5 ; 19,5 ; 18,2 ; 11,6$ and $1,2 \%$, were distributed in the wood of the stem, live branches, leaves, bark and branches dead, respectively. The accumulation of the elements in the tree was like this: leaves $(40,1 \%)$, live branches $(18,6 \%)$, bark $(17,9 \%)$, branches dead $(14,3 \%)$ and wood of the stem $(9,1 \%)$. The amount of elements contained in the above-biomass it was of $10,57 \mathrm{~kg}$ $\mathrm{ha}^{-1}$, accumulated in the following way: $\mathrm{Na}(50,6 \%), \mathrm{Fe}(23,3 \%), \mathrm{Zn}(12,5 \%), \mathrm{Mn}(7,8 \%), \mathrm{B}(4,4$ $\%)$ and $\mathrm{Cu}(1,4 \%)$. It is ended that $73,1 \%$ of the nutrients of the above-biomass of the acacia-black, they accumulated in the crown (leaves, live and dead branches).

Key words: Acacia mearnsii, black wattle, micronutrients, exportation of micronutrients and Na.

\footnotetext{
${ }^{1}$ Eng ${ }^{o}$. Florestal, M. Sc. Doutorando em Ciências Florestais / Universidade Federal do Paraná - Av. Pref. Lothário Meissner, 3400 - Jd. Botânico - 80210-170 - Curitiba, PR - e-mail: caldeira@ floresta.ufpr.br;

${ }^{2}$ Engo . Florestal, M. Sc. Prof. Não Titular / Universidade Estadual do Centro-Oeste, Doutorando em Ciências Florestais / Universidade Federal do Paraná - BR 153, km 7 - 84500-000 - Irati, PR - e-mail: rrondon@ floresta.ufpr.br;

${ }^{3}$ Eng ${ }^{\circ}$. Florestal, Dr. Prof. Adjunto do Departamento de Ciências Florestais / Universidade Federal de Santa Maria - 97105900 - Santa Maria, RS;

${ }^{4}$ Engo . Florestal, Dr. Prof. Visitante (Programa de Intercâmbio DAAD/CAPES. Universidade Federal de Maria. e-mail: peter.spathelf@forst.bwl.de.
} 


\section{INTRODUÇÃO}

A acácia-negra (Acacia mearnsii De Wild.) é uma espécie arbórea nativa da Austrália e começou a ser plantada comercialmente no Brasil, em 1935. Apresenta crescimento rápido e usos múltiplos. A madeira é utilizada na fabricação de celulose, aglomerado e energia. Da casca para a extração do tanino, empregado na indústria farmacêutica, coureira entre outras. Também é utilizada para a recuperação de solos degradados e em sistemas agroflorestais.

No Brasil é cultivada principalmente no Estado do Rio Grande do Sul, cobrindo cerca de 100.000 hectares. A maioria dessas plantações se encontram em pequenas propriedades rurais, com participação de mais de 10.000 produtores rurais (HIGA et al., 1998). Portanto, a cultura da acácianegra tem grande importância socioeconômica para esse Estado.

A maioria dos povoamentos de acácia-negra são implantados em solos com baixos níveis de fertilidade e as práticas de uso e manejo do solo geralmente são realizadas de forma incorreta. Sob tais condições, os índices de produtividade normalmente são baixos, sendo portanto, indispensável a adoção de práticas de manejo do solo florestal a fim de elevar os níveis de fertilidade e produtividade dos sítios (DALLAGO, 2000).

O conhecimento da distribuição dos nutrientes dentro das árvores é fundamental para estudar sua nutrição, reciclagem e exportação de nutrientes. Nesses aspectos, alguns trabalhos já foram realizados com a acácia-negra por CALDEIRA (1998); PEREIRA et al. (1999), (2000); CALDEIRA et al. (1999a), (1999b), (1999c), (2000). Entretanto, no que diz respeito a concentração e exportação de bioelementos na acácia-negra no Brasil, poucos trabalhos foram realizadas até então.

O objetivo do trabalho foi avaliar o conteúdo e a exportação de B, Cu, Fe, Mn, Zn e Na, nos componentes da biomassa acima do solo da acácia-negra, procedência australiana Lake Geoge Bunge Dore, com 2,4 anos de idade, plantada no município de Butiá - RS.

\section{MATERIAL E MÉTODOS}

O presente trabalho foi realizado em um povoamento de acácia-negra com 2,4 anos de idade e plantado no espaçamento 1,7 × 3,0 m, localizado na região fisionômica do Estado do Rio Grande do Sul, denominada Serra do Sudeste (Escudo Rio-grandense), município de Butiá - RS, situado entre as coordenadas geográficas $30^{\circ} 07^{\prime} 12^{\prime \prime} \mathrm{S}$ e $51^{\circ} 57^{\prime} 45^{\prime}$ " W e a uma altitude média de $35 \mathrm{~m}$ s.n.m.

Pelo sistema de classificação de Köppen o clima da região é do tipo Cfa, subtropical (MORENO, 1961). A temperatura média anual é de $18-19^{\circ} \mathrm{C}$, com temperaturas máximas e mínimas no ano de 24 e $14{ }^{\circ} \mathrm{C}$, respectivamente. A precipitação média anual é de $1.400 \mathrm{~mm}$ (IPAGRO, 1989). $\mathrm{O}$ solo da região pertence à Unidade de Mapeamento São Jerônimo, classificado como Argissolo Vermelho Escuro (EMBRAPA, 1999).

Para a quantificação dos nutrientes na biomassa acima do solo da acácia-negra selecionou-se nove árvores de procedência Lake George Bunge Dore, New South Wales, Austrália $\left(35^{\circ} 28^{\prime} \mathrm{S}\right.$ e $148^{\circ} 57^{\prime}$ E). Na parte intermediária da copa das árvores, nos quatro pontos cardeais, foram coletadas folhas para a realização de análise nutricional. Os galhos foram classificados como vivos e mortos, sendo que dos vivos todas as folhas foram colhidas.

A massa fresca total das folhas, galhos vivos e mortos, casca e madeira do tronco de todas as árvores foram determinadas no campo. De cada um desses componentes retirou-se amostras para aferição da massa fresca no campo. Posteriormente, cada componente foi acondicionado em sacos plásticos identificados e levados ao laboratório, onde foram embalados em sacos de papel pardo e colocados em estufa com circulação forçada $\left( \pm 75^{\circ} \mathrm{C}\right)$, até atingirem peso constante.

A madeira do tronco foi amostrada em um disco de 5,0 cm de espessura por árvore, retirado na metade da altura total da árvore (YONG \& CARPENTER, 1976), do qual separou-se casca e madeira, sendo registrado a massa fresca de cada componente. Após serem secos em estufa, ambos componentes foram picados para facilitar a moagem. Das amostras dos demais componentes (folhas, galho vivo e morto), foram moídas em moinho do tipo Wiley e passadas em peneira com malha de 1,0 $\mathrm{mm}$, retirou-se uma subamostra de cada componente para a realização das análises laboratoriais.

As determinações das concentrações dos elementos ( $\mathrm{B}, \mathrm{Cu}, \mathrm{Fe}, \mathrm{Mn}, \mathrm{Zn}$ e Na), em cada componente da árvore de acácia-negra, seguiu-se as metodologias propostas por TEDESCO et al. 
(1995). Já o estoque dos nutrientes avaliados em todos os componentes da biomassa acima do solo em $\mathrm{kg} \mathrm{ha}^{-1}$, foi obtido a partir da biomassa determinada por CALDEIRA (1998) e da concentração dos elementos em cada porção vegetal. A soma dos valores dos elementos estudados para cada componente da biomassa aérea forneceu a estimativa do conteúdo total em $\mathrm{kg} \mathrm{ha}^{-1}$ dos mesmos.

\section{RESULTADOS E DISCUSSÃO}

Observando a Figura 1, verifica-se que a biomassa acima do solo dos diferentes componentes das árvores de acácia-negra se encontra distribuída na seguinte ordem decrescente: lenho $>$ galhos vivos $>$ folhas >casca $>$ galhos mortos. Resultados muito semelhantes foram encontrados por CROMER et al. (1975), em Eucalyptus globulus com 4 anos de idade, sendo: madeira (55 \%), ramos $(17 \%)$, folhas $(16 \%)$ e casca (12\%). A produção total de biomassa acima do solo seca foi estimada em $41.107 \mathrm{~kg} \mathrm{ha}^{-1}$. CALDEIRA et al. (2000), pesquisando a ciclagem de nutrientes em um povoamento de acácia-negra, procedência Bodalla - Austrália, no mesmo local deste estudo e com idades iguais, estimaram uma produção $19.475 \mathrm{~kg} \mathrm{ha}^{-1}$ de biomassa aérea total, concentrada na seqüência: lenho $>$ folhas $>$ galho vivo $>$ casca $>$ galho morto. A grande discrepância de produção de biomassa aérea total pode ser atribuída ao fator genético das procedências, pois ambas foram estabelecidas nas mesmas condições as edafo-climáticas e receberam práticas idênticas de implantação e manejo.

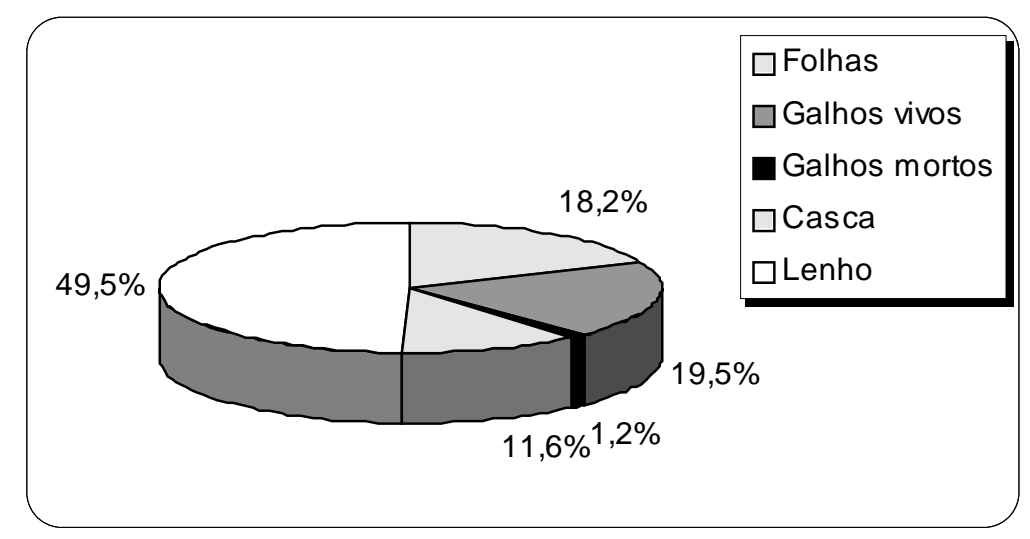

Figura 1: Distribuição da biomassa acima do solo da acácia-negra (Acacia mearnsii De Wild.) procedência australiana Lake Geoge Bunge Dore, com 2,4 anos de idade no município de Butiá - RS.

Ao observar os dados apresentados na Tabela 1, verifica-se que os resultados do conteúdo, quantidade média e proporção dos elementos acumulados em cada componente da biomassa acima do solo da acácia-negra, procedência Lake George Bunge Dore - Austrália. Foi verificado que 40,1 \% dos 
Tabela 1: Resultados do conteúdo $\left(\mathrm{mg} \mathrm{kg}^{-1}\right)$, quantidade média $\left(\mathrm{kg} \mathrm{ha}^{-1}\right)$, proporção (\%) de $\mathrm{B}, \mathrm{Cu}, \mathrm{Fe}, \mathrm{Mn}, \mathrm{Zn}$ e Na, nos diferentes componentes da biomassa aérea de acácia-negra (Acacia mearnsii De Wild.) procedência australiana Lake George Bunge Dore, com 2,4 anos de idade no município de Butiá-RS.

\section{COMPONENT $\quad$ Elementos (Elements)}

ES

\begin{tabular}{|c|c|c|c|c|c|c|c|c|c|c|c|c|c|c|c|c|c|c|}
\hline \multirow{3}{*}{ (Components) } & \multirow{2}{*}{\multicolumn{3}{|c|}{ B }} & \multirow{2}{*}{\multicolumn{3}{|c|}{$\mathrm{Cu}$}} & \multirow{2}{*}{\multicolumn{3}{|c|}{$\mathrm{Fe}$}} & \multirow{2}{*}{\multicolumn{3}{|c|}{ Mn }} & \multirow{2}{*}{\multicolumn{3}{|c|}{$\mathbf{Z n}$}} & \multirow{2}{*}{\multicolumn{3}{|c|}{$\mathrm{Na}$}} \\
\hline & & & & & & & & & & & & & & & & & & \\
\hline & $\mathrm{mg} \mathrm{kg}^{-1}$ & $\mathrm{~kg} \mathrm{ha}^{-1}$ & $\%$ & $\mathrm{mg} \mathrm{kg}^{-1}$ & $\mathrm{~kg} \mathrm{ha}^{-1}$ & $\%$ & $\mathrm{mg} \mathrm{kg}^{-1}$ & $\mathrm{~kg} \mathrm{ha}^{-1}$ & $\%$ & $\mathrm{mg} \mathrm{kg}^{-1}$ & $\mathrm{~kg} \mathrm{ha}^{-1}$ & $\%$ & $\mathrm{mg} \mathrm{kg}^{-1}$ & $\mathrm{~kg} \mathrm{ha}^{-1}$ & $\%$ & $\mathrm{mg} \mathrm{kg}^{-1}$ & $\mathrm{~kg} \mathrm{ha}^{-1}$ & $\%$ \\
\hline $\begin{array}{l}\text { Folhas } \\
\text { (Leaves) }\end{array}$ & 18,78 & 0,141 & 1,334 & 8,06 & 0,060 & 0,568 & 147,89 & 1,107 & 10,476 & 51,89 & 0,388 & 3,672 & 121,22 & 0,907 & 8,583 & 236,33 & 1,769 & 16,741 \\
\hline $\begin{array}{l}\text { Galhos vivos } \\
\text { (Live branches) }\end{array}$ & 15,56 & 0,125 & 1,183 & 2,89 & 0,023 & 0,218 & 71,67 & 0,574 & 5,432 & 8,93 & 0,071 & 0,672 & 22,00 & 0,176 & 1,666 & 149,56 & 1,198 & 11,337 \\
\hline $\begin{array}{l}\text { Galhos } n \\
\text { (Dead bi }\end{array}$ & 9,22 & 0,005 & 0,047 & 2,43 & 0,001 & 0,009 & 83,22 & 0,043 & 0,407 & 12,27 & 0,006 & 0,057 & 17,22 & 0,009 & 0,085 & 84,11 & 0,043 & 0,407 \\
\hline $\begin{array}{l}\text { Casca } \\
\text { (Bark) }\end{array}$ & 19,22 & 0,091 & 0,861 & 2,54 & 0,012 & 0,114 & 95,56 & 0,454 & 4,296 & 38,00 & 0,180 & 1,703 & 19,78 & 0,094 & 0,890 & 84,78 & 0,403 & 3,814 \\
\hline $\begin{array}{l}\text { Madeira } \\
\text { (Wood) }\end{array}$ & 4,89 & 0,099 & 0,937 & 2,62 & 0,053 & 0,502 & 13,78 & 0,280 & 2,650 & 8,78 & 0,179 & 1,694 & 6,81 & 0,139 & 1,315 & 95,22 & 1,937 & 18,331 \\
\hline Total & 67,67 & 0,461 & 4,363 & 18,54 & 0,149 & 1,410 & 412,12 & 2,458 & 23,261 & 119,87 & 0,824 & 7,798 & 187,03 & 1,325 & 12,54 & 650,00 & 5,350 & 50,629 \\
\hline
\end{tabular}


elementos acumulados estavam presentes nas folhas, 18,6 \% nos galhos vivos, $17,9 \%$ na casca, 14,3 $\%$ nos galhos mortos e 9,1 \% no lenho da madeira. Num povoamento de Eucalyptus grandis com 2,5 anos de idade, POGGIANI et al. (1983) observaram que do total de micronutrientes ( $\mathrm{Cu}, \mathrm{Fe}, \mathrm{Mn}, \mathrm{Zn}$ ), $23,0 \%$ se concentravam nas folhas, $6,8 \%$ nos galhos, $29,9 \%$ na copa e 40,16 \% na madeira. Segundo KRAMER \& KOZLOWSKI (1979) o elevado conteúdo de nutrientes nas folhas é devido a concentração da maioria das células vivas da árvore neste compartimento da árvore, que tendem a acumular maiores quantidades de nutrientes, em função dos processos de transpiração e fotossíntese.

A estimativa do estoque dos nutrientes estudados em todos os componentes da biomassa acima do solo da acácia-negra foi de $10,57 \mathrm{~kg} \mathrm{ha}^{-1}$. Desse total, a contribuição dos elementos analisados na biomassa total foi a seguinte: $\mathrm{Na}(50,6 \%), \mathrm{Fe}(23,3 \%), \mathrm{Zn}(12,5 \%), \mathrm{Mn}(7,8 \%), \mathrm{B}(4,4$ $\%)$ e $\mathrm{Cu}(1,4 \%)$. Valores semelhantes de nutrientes na biomassa aérea foram encontrados por BAGGIO (1994), em cinco florestas naturais de bracatinga (Mimosa scabrella Benth.), situados na região metropolitana de Curitiba - PR, onde estimou uma produção média de $10,06 \mathrm{~kg} \mathrm{ha}^{-1}$, com a seguinte contribuição dos nutrientes: $\mathrm{Mn}(55,7 \%), \mathrm{Fe}(34,7 \%), \mathrm{Zn}(2,6 \%)$ e Cu $(2,1 \%)$.

As maiores concentrações dos nutrientes analisados se encontram nas folhas da acácia-negra com as respectivas participações: $\mathrm{Zn}(69,0 \%), \mathrm{Mn}(47,0 \%), \mathrm{Cu}(41,0 \%), \mathrm{Fe}(46,0 \%), \mathrm{B}(31,0 \%)$, exceto o $\mathrm{Na}(36,2 \%)$ que apresentou maior conteúdo no lenho da madeira. Na copa (folhas e galhos vivos e mortos) se concentram $73,1 \%$ do total dos nutrientes acumulados na biomassa, respectivamente. Os elevados conteúdos de bioelementos nas folhas, torna esse componente vegetal importante na ciclagem de nutrientes, embora represente pequena porção da biomassa total (Figura 1). Tal fato, também foi observado por CALDEIRA et al. (1999a), para os macronutrientes da acácianegra de procedência e idades iguais à do presente estudo.

Os componentes da árvores que normalmente são explorados do povoamento, ou seja, a casca e o lenho da madeira contribuíram com 17,9 e 9,1\%, respectivamente, totalizando cerca de $26,9 \%$ do total de elementos acumulados, lembrando que a rotação da acácia-negra ocorre entre 7 e 9 anos. Valores superiores foram encontrados por POGGIANI et al. (1983), em um povoamento Eucalyptus grandis com 2,5 anos de idade, onde cerca de $40 \%$ dos micronutrientes são exportados por ocasião da retirada da madeira.

\section{CONCLUSÕES}

Dos elementos estudados na biomassa acima do solo da acácia-negra $73,1 \%$ são acumulados na copa (folhas e galhos verdes e mortos). Sistemas de exploração que deixam folhas e galhos no sítio contribuem para a ocorrência de menores exportações dos bioelementos. No entanto, o restante dos elementos que se encontram acumulados na casca e no lenho da madeira são os produtos exportados do sítio.

\section{AGRADECIMENTOS}

Os autores manifestam seus agradecimentos ao Engenheiro Florestal Elias Moreira dos Santos - Supervisor de Pesquisa e Desenvolvimento da Agroseta S. A, pela indispensável e árdua colaboração nas atividades de campo.

\section{REFERÊNCIAS BIBLIOGRÁFICAS}

BRASIL. Ministério da Agricultura - Departamento de Pesquisa Agropecuária. Levantamento de reconhecimento dos solos do Estado do Rio Grande do Sul. Recife, 1973. 431p. (Boletim Técnico, 30).

BAGGIO, A.J. Estudio sobre el sistema agroforestal tradicional de la bracatinga (Mimosa scabrella Benth.) en Brasil productividad, manejo de residuos y elaboracion de compost. Madrid: Escuela Tecnica Superior de Ingenieros de Montes, 1994. 242p. (Tese - Doutorado).

CALDEIRA, M.V.W.; SCHUMACHER, M.V.; TEDESCO, N.; SANTOS, E.M. dos. Ciclagem de nutrientes em Acacia mearnsii De Wild. V quantificação do conteúdo de nutrientes na biomassa 
aérea de Acacia mearnsii De Wild. procedência australiana. Ciência Rural, Santa Maria, v.30, n.6, p.977-982, 2000.

CALDEIRA, M.V.W.; SCHUMACHER, M.V.; SANTOS, E.M. dos.; TEDESCO, N.; PEREIRA, J.C. Estimativa do conteúdo de nutrientes em um povoamento jovem de Acacia mearnsii De Wild. estabelecido na região sul do Brasil. Floresta, Curitiba, v.29, n.1 e 2, p.53-65, 1999a.

CALDEIRA, M.V.W.; SCHUMACHER, M.V.; PEREIRA, J.C.; DELLAFLORA, J.B; SANTOS, E.M. dos. Concentração e redistribuição de nutrientes nas folhas e no folhedo em povoamento comercial de Acacia mearnsii De Wild. plantado no Rio Grande do Sul. Ciência Florestal, Santa Maria, v.9, n.1, p.19-24, 1999b.

CALDEIRA, M.V.W.; PEREIRA, J.C.; SCHUMACHER, M.V.; DELLAFLORA, J.B; SANTOS, E.M. dos. Comparação entre as concentração de nutrientes nas folhas e no folhedo em procedências de Acacia mearnsii De Wild. Rev. Árvore, Viçosa, v.23, n.4, p.489-492, 1999c.

CALDEIRA, M.V.W. Quantificação da biomassa e do conteúdo de nutrientes em diferentes procedências de acácia-negra (Acacia mearnsii De Wild.). Santa Maria: Universidade Federal de Santa Maria (UFSM), 1998. 96p. (Dissertação - Mestrado em Engenharia Florestal).

CROMER, R.N.; RAUPACH, M.; CLARKE, A.R.P.; CAMERON, J.N. Eucalyptus plantations in Australia - the potential for intensive production. Appita, v.29, p.165-173, 1975.

DALLAGO, J.S. Utilização da cinza de biomassa de caldeira como fonte de nutrientes no crescimento de plantas de acácia-negra (Acacia mearnsii De Wild.). Santa Maria: Universidade Federal de Santa Maria (UFSM), 2000. 64p. (Dissertação - Mestrado em Engenharia Florestal).

EMBRAPA - Empresa Brasileira de Pesquisa Agropecuária. Sistema brasileiro de classificação de levantamento de solos. EMBRAPA: SNPS. Rio de Janeiro, 1999.

HIGA, A.R.; DEDECEK, R.A.; SANTOS, A.F. dos; STEIN, P.P.; SIMON, A.A. Desarrollo de sistemas de produccoón para acacia negra (Acacia mearnsii De Wild.). In: CONGRESSO LATINOAMERICANO IUFRO - O manejo sustentable de los recursos forestales, desafío del siglo XXI, 1., 1998, Valdivia. CD-Rom ... Valdivia: IUFRO, 1998.

IPAGRO - Instituto de Pesquisas Agronômicas. Atlas agroclimático do Estado do Rio Grande do Sul. Porto Alegre, 1989. 3v.

KRAMER, P.J.; KOZLOWSKI, T.T. Physiology of wood plants. New York: Academic Press, 1979. $811 \mathrm{p}$.

MORENO, J.A. Clima do Rio Grande do Sul. Porto Alegre: Secretaria da Agricultura, 1961. 42p.

PEREIRA, J.C.; CALDEIRA, M.V.W.; SCHUMACHER, M.V.; HOPPE, J.M.; SANTOS, E.M. dos. Exportação de nutrientes em um povoamento de Acacia mearnsii De Wild. em idade de corte. In: CICLO DE ATUALIZAÇÃO FLORESTAL DO CONE-SUL, 1. Santa Maria - RS. Anais... Santa Maria: UFSM, 1999. p.158-164.

PEREIRA, J.C.; CALDEIRA, M.V.W.; SCHUMACHER, M.V.; HOPPE, J.M. SANTOS, E.M. dos. Estimativa do conteúdo de nutrientes em um povoamento de de Acacia mearnsii De Wild. no Rio Grande do Sul - Brasil. Rev. Árvorev.24, n.2, p.193-199, 2000.

POGGIANI, F.; COUTO, H.T.Z.; SOITER-FILHO, W. Biomass and nutrient estimates removal in short rotation intensively cultured plantations on Eucalyptus grandis. IPEF, Piracicaba, v.23, p.3742, 1983

TEDESCO, M.J.; GIANELLO, C.; BISSANI, C.A.; BOHNEN, H.; VOLKWEISS, S.J. Análise de solos, plantas e outros materiais. Porto Alegre: UFRGS - Departamento de Solos/Faculdade de Agronomia, 1995. 174p. (Boletim Técnico, 5).

YONG, H.E.; CARPENTER, P.N. Sampling variation of nutrient element content within and between on trees of the sama species. In: OSLO BIOMASS STUDIES, 1976, Oslo. Proceedings ... Oslo, 1976. p.75-90. 